\title{
PLASTICIDADE FENOTIIPICO-VEGETATIVA DE Oeceoclades maculata (LINDL.) LINDL. (ORCHIDACEAE) EM DOIS AMBIENTES DE ÁREA FLORESTADA
}

\author{
Michele Stencel $^{*}$ \\ Renato Luis Bertin* \\ Thiago Souza-Leal ${ }^{*}$ \\ Cristiano Pedroso-de-Moraes ${ }^{* * *}$
}

RESUMO: A plasticidade fenotípica é a habilidade genotípica de produzir fenótipos diferenciados em resposta a diferentes condições ambientais. Oeceoclades maculata, orquídea invasora, apresenta elevada capacidade adaptativa relacionada à colonização de diferentes babitat, especialmente os antropizados. Dessa forma, o presente trabalho teve como objetivo realizar análises morfobiométricas nesta espécie, a fim de evidenciar adaptações plástico-fenotípico-vegetativas em dois ambientes lumínicos ocorrentes no Arboreto de Essências Nativas, em Araras (SP). Para as análises foram coletados 50 indivíduos de Oeceoclades maculata, em maturidade fisiológica, tanto na borda, quando no interior do fragmento. Foram inferidos os seguintes dados biométricos: número médio de raízes (NMR), pseudobulbos (NMP) e folhas (NMF), comprimento médio de raízes (CMR), pseudobulbos (CMP) e folhas (CMF), o diâmetro médio de raízes (DMR) e pseudobulbos (DMP), largura (LMF) e espessura média foliar (EMF), massa fresca de raízes (MFR), pseubolbulbos (MFP) e folhas (MFF) e de massa seca de raízes (MSR), pseubolbulbos (MSP) e folhas (MSF). $\mathrm{O}$ índice de plasticidade fenotípica (IPF) foi calculado para todas as variáveis morfobiométricas obtidas. As análises morfobiométricas evidenciaram adaptações plástico-fenotípico-vegetativas em indivíduos de Oeceoclades maculata viventes nos diferentes ambientes do Arboreto de Essências Nativas. Tais adaptações estão correlacionadas principalmente às condições lumínicas ocorrentes na borda e no interior do fragmento.

PALAVRAS-CHAVE: Adaptação; Fenótipo; Morfobiometria; Orquídeas.

\footnotetext{
Discentes de Ciências Biológicas no Centro Universitário Herminio Ometto de Araras (UNIARARAS), Brasil.

** Mestrado em Ciências Biológicas pela Universidade Estadual Paulista Júlio de Mesquita Filho (UNESP), Rio Claro (SP), Brasil.

*** Docente Doutor em Biologia Vegetal; Docente no Centro Universitário Hermínio Ometto (UNIARARAS), Araras, (SP), Brasil; E-mail: pedroso@uniararas.br
} 


\section{PHENOT YPIC AND VEGETATIVE PLASTICITY OF Oeceoclades maculata (LINDL.) LINDL. (ORCHIDACEAE) IN T WO ENVIRONMENTS OF A FOREST AREA}

ABSTRACT: Phenotypic plasticity is the genotypic capacity in producing different phenotypes as a response to different environmental conditions. The invading orchid Oeceoclades maculata has high adaptative capacity related to the colonization of different habitats, particularly, anthropic ones. Current paper provides morphobiometric analyses of the species to report plastic-phenotypic-vegetative adaptations in two luminic environments in the Arboreto de Essências Nativas, in Araras SP Brazil. Fifty specimens of Oeceoclades maculata, featuring physiological maturity, were collected from the edge and the interior of the forest fragment. The following biometric data were taken: mean number of roots and pseudobulbs, leaves, mean length of roots and pseudobulbs, leaves, mean diameter of leaves and pseudobulbs, mean length and thickness of leaves, fresh mass of roots and pseudobulbs and leaves, dry mass of roots and pseudobulbs and leaves. Phenotypic plasticity index was calculated for all morphobiometric variables. Morphobiometric variables revealed plastic-phenotypic-vegetative adaptations in Oeceoclades maculata specimens in different environments of the Arboreto de Essências Nativas. Adaptations are mainly co-related to luminic conditions on the fragment's edge and interior.

KEY WORDS: Adaptation; Phenotype; Morphobiometry; Orchids.

\section{INTRODUÇÃO}

Representantes de Orchidaceae são sensíveis a interferências antrópicas em matas, devido, principalmente, a ocuparem nichos muito especializados nos ambientes em que vivem (ZOTZ; ANDRADE, 2002). Contudo, existem espécies de orquídeas que se adaptaram a essas alterações, sendo consideradas invasoras de ambientes perturbados (ACKERMAN, 2007; COHEN; ACKERMAN, 2009; LIU; PEMBERTON, 2010), sendo capazes de sobreviver em tais áreas devido à sua ampla capacidade adaptativa intrinsecamente relacionada à capacidade de gerar diferenciados fenótipos.

O fenômeno da plasticidade fenotípica pode ser entendido como a habilidade genotípica de produzir fenótipos diferenciados em resposta a diferentes 
condições ambientais, sendo este um dos meios pelos quais as plantas podem alterar sua morfologia e fisiologia, a fim de enfrentar constantes modificações, sejam elas sazonais naturais (GIANOLI, 2004; PIGLIUCCI, 2001; SMEKENS; VAN TIENDEREN, 2001) ou antrópicas (MORAES; ALMEIDA, 2004).

Teoriza-se que espécies com distribuição ecológica restrita evidenciam menor tolerância a variações ambientais ao contrário das generalistas, as quais apresentam maior distribuição geográfica, sendo capazes, portanto, de resistir a grandes variações em seu babitat (FUTUYMA; MORENO, 1988; GONZÁLEZ; GIANOLI, 2004). Tal evidência pode ser comprovada pelo fato de que em condições naturais, fatores abióticos como luz e água variam espacial e temporalmente em diferentes ambientes, restringindo o crescimento e a distribuição das plantas (SCHULZE et al., 1987; SULTAN; BAZZAZ, 1993). Nesse ínterim, estudos demonstram que, para várias espécies, as variações na disponibilidade de recursos podem ocasionar mudanças morfológicas e alterações na assimilação de biomassa (PALACIO-LÓPEZ; RODRÍGUEZ-LÓPEZ, 2007; VALLADARES et al., 2002).

Oeceoclades maculata (Lindl.) Lindl. apresenta elevada capacidade adaptativa relacionada à colonização de diferentes babitat, especialmente os antropizados (COHEN; ACKERMAN, 2009). Após ser introduzida, acabou se naturalizando no continente americano (FELDMANN; BARRÉ, 2001), alcançando ampla distribuição, estando presente por todo o Brasil, Colômbia, Venezuela e América Central, chegando até a Flórida (STERN, 1988). Nacionalmente, tal espécie pode ser encontrada em diversas fitofisionomias, com condições climáticas variadas, como a Mata Estacional Semidecidual (KINOSHITA et al., 2006; PANSARIN; PANSARIN, 2008), o Cerrado (BATISTA; BIANCHETTI, 2003; BATISTA et al., 2005) e a Caatinga (ARAÚJO et al., 2005), ocorrendo na maioria das vezes em matas ribeirinhas destes biomas (ARAÚJO et al., 2005; BUENO; RESENDE; GOMES, 2007; MENINI-NETO; ALMEIDA; FORZZA, 2004;). Ainda, Ferreira, Lima e Pansarin (2010), em levantamento realizado na região central de São Paulo, catalogaram a espécie em todas as fitofisionomias estudadas. Para Mogi Guaçu, a espécie foi registrada em área de intensa atividade antrópica (PEDROSO-DE-MORAES et al., 2010), aparecendo também em Mogi Mirim, município limítrofe, em área natural remanescente (SCHUSTER et al., 2010). Entretanto, mesmo com esta distribuição fitogeográfica, 
esta carece de estudos morfofisiológicos mais aprofundados, pois além de sua importância ecológica, esta, ainda, apresenta-se potencialmente fitoterápica, tendo sido encontrado para espécie atividade antioxidante em extrato hidroalcóolico bruto a frio (CARRERA et al., 2006). Dessa forma, o presente trabalho apresentou como objetivo realizar análises morfobiométricas em indivíduos de $O$. maculata a fim de evidenciar adaptações plástico-fenotípico-vegetativas em dois ambientes lumínicos ocorrentes no Arboreto de Essências Nativas do Centro Universitário Hermínio Ometto - Uniararas, em Araras (SP).

\section{MATERIAL E MÉTODOS}

\subsection{CARACTERIZAÇÃO MORFOLÓGICA DA ESPÉCIE}

Oeceoclades maculata é uma espécie terrestre humícola de porte herbáceo, sempre verde, com crescimento simpodial. Os pseudobulbos são verdes, piriformes, com cerca de $2 \mathrm{~cm}$ de comprimento, 1-foliados, envoltos por bainhas, bainhas caulinares lanceoladas, $4-6 \times 3 \mathrm{~cm}$, paleáceas, ápice agudo. Folha apical, bainha foliar com cerca de 1 x $0,7 \mathrm{~cm}$ de largura, lâmina verde-clara pintalgada de verde-escuro, elíptica, 10-12,5 x 2,5-3 cm, conduplicada, coriácea, margem inteira, ápice agudo. A inflorescência é em racemo com $26-31 \mathrm{~cm}$ de comprimento, pauciflora, ereta, laxa, terminal. Pedúnculo $22-25 \mathrm{~cm}$ de comprimento, ereto, brácteas do pedúnculo lanceoladas, 2,3-3,2 x 0,5 cm, membranáceas, amplectivas sobre o pedúnculo, ápice agudo, brácteas florais triangulares, cerca de 4 × $2 \mathrm{~mm}$, membranáceas, ápice agudo. Flores pediceladas, calcaradas, pedicelo com cerca de $3 \mathrm{~mm}$ de comprimento, sépala dorsal suboblanceolada, cerca de $2 \times 9 \mathrm{~mm}$, côncava, ápice agudo, sépalas laterais subfalciformes, cerca de $8 \times 2 \mathrm{~mm}$, ápice agudo, pétalas elípticas, cerca de 8 x $3 \mathrm{~mm}$, subassimétricas, aderentes à sépala dorsal, ápice agudo, labelo trilobado, cerca de $7 \mathrm{~mm} \times 8 \mathrm{~mm}$, lobos laterais cerca de $2 \times 3 \mathrm{~mm}$, ápice arredondado, lobo central bilobulado, lóbulos suborbiculares cerca de $3 \times 3 \mathrm{~mm}$, dois calos na base do labelo, cálcar cilíndrico, cerca de $4 \mathrm{~mm}$ de comprimento, coluna com cerca de 5 $\mathrm{mm}$ de comprimento, rostro triangular no ápice, cerca de $1 \mathrm{~mm}$ de comprimento, 
4 polínias, ovário de cerca de $1 \mathrm{~cm}$ de comprimento. Frutos maduros, amarelados, cerca de $2,3 \mathrm{~cm}$ de comprimento, perianto persistente (MENINI-NETO; ALMEIDA; FORZZA, 2004).

\subsection{MATERIAL BOTÂNICO}

O trabalho foi desenvolvido de janeiro a setembro de 2013, no Arboreto e no Laboratório de Botânica e Análises Ambientais do Centro Universitário Hermínio Ometto - Uniararas, município de Araras (SP). O arboreto caracteriza-se como área florestada no ano de 1996, implantada com essências nativas oriundas de Floresta Estacional Semidecidual dentro do campus universitário, estando sob as coordenadas $22^{\circ} 22^{\prime} 31.00^{\prime \prime S}$ e $47^{\circ} 22^{\prime} 04.29^{\prime \prime} \mathrm{O}$, elevavação de $652 \mathrm{~m}$ e perfazendo uma área aproximada de $9.300 \mathrm{~m}^{2}$.

A região de Araras encontra-se sob clima tropical, sazonal, com verão chuvoso e inverno seco, descrito como Cwa na classificação climática de Köppen. As chuvas não ultrapassam $30 \mathrm{~mm}$ durante o mês mais seco e a temperatura do mês mais quente oscila entre $19^{\circ} \mathrm{C} \mathrm{e} 29^{\circ} \mathrm{C}$ (CEPAGRI, 2007). Nesta região há predomínio do Latossolo Vermelho-Amarelo, com manchas mais férteis de Latossolo Vermelho Escuro (PIVELLO-POMPÉIA, 1985). Especificamente em relação ao microclima da área de estudo, para a borda com relação à intensidade luminosa, umidade relativa do ar e temperatura foram obtidos os seguintes dados médios anuais: $1420 \mu \mathrm{mol} . \mathrm{m}$ ${ }^{2} \cdot s^{-1}, 37,5 \%$ e $38,6{ }^{\circ} \mathrm{C}$ e para o interior do fragmento de $540 \mu$ mol. $^{-2} \cdot s^{-1}, 82,3 \%$ e 21,3 ${ }^{\circ} \mathrm{C}$, respectivamente.

\subsection{ANÁLISES MORFOBIOMÉTRICAS E FENOTÍPICAS}

Para a realização das análises morfométricas e fenotípicas foram coletados 50 indivíduos de Oececlades maculata, em maturidade fisiológica, em quatro pontos distantes (10 metros) tanto na borda, com distância máxima de até $20 \mathrm{~m}$ em direção ao interior, quando no interior do fragmento (mais de $50 \mathrm{~m}$ a partir da borda) (WILLIAMS-LINERA, 1990).

Foram inferidos os seguintes dados biométricos: número médio de raízes (NMR), pseudobulbos (NMP) e folhas (NMF), comprimento médio de raízes (CMR), 
dos pseudobulbos (CMP) e das folhas (CMF), o diâmetro médio de raízes (DMR) e dos pseudobulbos (DMP), largura (LMF) e espessura média foliar (EMF), massa fresca de raízes (MFR), dos pseudolbulbos (MFP) e das folhas (MFF) e a massa seca de raízes (MSR), pseudolbulbos (MSP) e folhas (MSF) tanto nos indivíduos coletados na borda, quanto no interior do fragmento.

$O$ índice de plasticidade fenotípica (IPF) foi calculado para todas as variáveis morfobiométricas obtidas (VALLADARES et al., 2000), onde: IPF = (máximomínimo) /máximo. Os valores de IPF serão calculados com base nas médias obtidas. Os resultados obtidos de IPF nos dois ambientes foram submetidos à análise da variância, seguida de comparações de médias pelo teste de Tukey em nível de 5\% de probabilidade realizados por meio do pacote estatístico BioEstat 5.3.

\section{RESULTADOS E DISCUSSÃO}

Os resultados relativos aos índices médios de plasticidade fenotípica obtidos após a análise estatística demonstraram que em relação ao sistema radicial e caulinar, as plantas presentes no interior do arboreto apresentaram maiores valores para as variáveis comprimento (CMR), diâmetro (DMR) e massa fresca (MFR), enquanto as plantas situadas na borda apresentaram maiores valores para massa seca (MSR) e número médio de raízes (NMR) e pseudobulbos (NMP). Com relação aos resultados obtidos para as folhas, puderam ser constatados maiores valores médios no interior do arboreto para as variáveis comprimento (CMF), massa fresca (MFF) e número médio de folhas (NMF), enquanto valores mais elevados foram obtidos na borda para espessura (EMF), largura (LMF) e massa seca foliar (MSF) (Tabela 1).

Tabela 1. Índices de plasticidade fenotípica obtidos de indivíduos de Oeceoclades maculata ocorrentes na borda e no interior do Arboreto de Essências Nativas Uniararas, Araras (SP)

(Continua)

\begin{tabular}{ccc}
\hline Variáveis & Borda & Interior \\
\hline & Raizes & \\
\hline CMR & $0.067 \mathrm{~b}^{*}$ & $0.147 \mathrm{a}$ \\
DMR & $0.017 \mathrm{~b}^{*}$ & $0.117 \mathrm{a}$ \\
MFR & $0.089 \mathrm{~b}^{*}$ & $0.943 \mathrm{a}$ \\
\hline
\end{tabular}


(conclusão)

\begin{tabular}{|c|c|c|}
\hline Variáveis & Borda & Interior \\
\hline \multicolumn{3}{|c|}{ Raízes } \\
\hline MSR & $0.018 \mathrm{a}^{*}$ & $0.009 \mathrm{~b}$ \\
\hline NMR & $0.312 \mathrm{a}^{*}$ & $0.157 \mathrm{~b}$ \\
\hline \multicolumn{3}{|c|}{ Pseudobulbos } \\
\hline CMP & $0.072 b^{*}$ & $0.612 \mathrm{a}$ \\
\hline DMP & $0.102 b^{*}$ & $0.227 \mathrm{a}$ \\
\hline MFP & $0.168 b^{*}$ & $0.213 \mathrm{a}$ \\
\hline MSP & $0.099 \mathrm{a}^{*}$ & $0.023 \mathrm{~b}$ \\
\hline NMP & $0.407 \mathrm{a}^{*}$ & $0.292 \mathrm{~b}$ \\
\hline \multicolumn{3}{|c|}{ Folbas } \\
\hline CMF & $0.122 \mathrm{~b}^{* *}$ & $0.132 \mathrm{a}$ \\
\hline EMF & $0.332 \mathrm{a}^{*}$ & $0.112 \mathrm{~b}$ \\
\hline LMF & $0.242 \mathrm{a}^{*}$ & $0.132 \mathrm{~b}$ \\
\hline MFF & $0.213 b^{*}$ & $0.323 \mathrm{a}$ \\
\hline MSF & $0.142 \mathrm{a}^{*}$ & $0.091 \mathrm{~b}$ \\
\hline NMF & $0.222 b^{*}$ & $0.382 \mathrm{a}$ \\
\hline
\end{tabular}

Letras iguais na mesma linha não diferente entre si pelo teste de Tukey. * significante a $\mathrm{p}>0,01 ; * *$ significante a $\mathrm{p}>0,05 . \mathrm{CMR}=$ comprimento médio radicial $(\mathrm{cm}) ; \mathrm{DMR}=$ diâmetro médio radicial $(\mathrm{cm}) ;$ MFR $=$ massa fresca radicial $(\mathrm{g}) ; \mathrm{MSR}=$ massa seca radicial $(\mathrm{g}) ; \mathrm{NMR}=$ número médio radicial; $\mathrm{CMP}=$ comprimento médio do pseudobulbo $(\mathrm{cm}) ; \mathrm{DMP}=$ diâmetro médio do pseudobulbo $(\mathrm{cm}) ; \mathrm{MFP}=$ massa fresca do pseudobulbo (g); MSP = massa seca do pseudobulbo (g); NMP = número médio de pseudobulbos; $\mathrm{CMF}=$ comprimento médio foliar $(\mathrm{cm})$; $\mathrm{EMF}=$ espessura média foliar $(\mathrm{cm}) ; \mathrm{LMF}=$ largura média foliar $(\mathrm{cm}) ; \mathrm{MFF}=$ massa fresca foliar $(\mathrm{g}) ; \mathrm{MSF}=$ massa seca foliar $(\mathrm{g}) ; \mathrm{NMR}=$ número médio foliar.

Durante décadas acreditou-se que a plasticidade fenotípica limitaria as novidades evolutivas por reduzir as pressões seletivas em estruturas genéticas populacionais (WRIGHT, 1931). Entretanto, agora, sabe-se que a plasticidade é considerada como um mecanismo gerador de variabilidade e, como a seleção natural age sobre fenótipos, criam-se oportunidades para que mutações genéticas adaptativas ocorram. Ainda, se divergências fenotípicas geradas dentro de uma população forem mantidas por seleção disruptiva, haverá favorecimento para o surgimento de subespécies ou ecótipos (CARDOSO; LOMÔNACO, 2003; THOMPSON, 1991; VIA; LANDE, 1985).

As diferenças fenotípicas encontradas para plantas de Oeceoclades maculata crescentes na borda e no interior do fragmento corroboram com a sugestão de 
que uma população que ocupe um ambiente heterogêneo apresenta grande potencial plástico em suas características fisiológicas e/ou morfológicas (FUZETO; LOMÔNACO, 2000), assim, os resultados obtidos para MSR, NMR, MSP, NMP, EMF, LMF e MSF demonstram que os indivíduos crescentes sob maior irradiância (média anual de $1420 \mu \mathrm{mol} \cdot \mathrm{m}^{-2} \cdot \mathrm{s}^{-1}$ ), como na borda do fragmento analisado, apresentam balanço de carbono superior aos indivíduos presentes no interior da mata (média anual de $540 \mu \mathrm{mol} \cdot \mathrm{m}^{-2} \cdot \mathrm{s}^{-1}$ ), possibilitando melhores condições para crescimento e desenvolvimento. Nesse ínterim, em florestas tropicais, a luz, principalmente no caso de organismos fotossintéticos sésseis, mostra-se como o fator ambiental mais importante, em relação ao estabelecimento, crescimento e sobrevivência das plantas (POORTER, 2001; SULTAN, 1993; VALLADARES et al., 1997). Espécies sucessivas pioneiras/invasoras como Oeceoclades maculata (ACKERMAN, 2007; COHEN; ACKERMAN, 2009), geralmente apresentam elevada capacidade plástica, sendo potencialmente capazes de aclimatar-se às variações de luminosidade, podendo alterar a morfologia de seus órgãos, principalmente folhas, em resposta a diferentes níveis de irradiância (VOLTAN; FAHL; CARELLI, 1992).

Espécies vegetais crescendo sob elevada intensidade luminosa desenvolvem folhas mais curtas a fim de limitar a transpiração, e espessas, pelo desenvolvimento de muitas camadas no mesófilo (LARCHER, 2003). Tais modificações plásticas refletem a necessidade de maior eficiência nos processos fotossintéticos devido à elevação da taxa metabólica influenciada pela radiação luminosa (KERBAUY, 2008; LARCHER, 2003; TAIZ; ZEIGER, 2004) e culminam em maiores valores de massa seca e número de órgãos (LARCHER, 2003), assim, como encontrado neste trabalho para raízes, caules (pseudobulbos) e folhas. Tal constatação também é corroborada pelo fato de que os diferentes processos de crescimento e desenvolvimento que ocorrem ao mesmo tempo em uma planta estão intrinsecamente relacionados, podendo o desenvolvimento de uma parte afetar de forma positiva ou negativa $o$ da outra (CUZZUOL et al., 1996). Tal comportamento é bem evidenciado durante a formação de sistemas radiciais, para o qual o crescimento acelerado pode afetar negativamente ou até mesmo inibir o desenvolvimento caulinar e foliar. Entretanto, esta relação se deve ao fato de que o crescimento ativo do sistema radicial necessita de substâncias orgânicas translocadas das folhas para a base, podendo implicar no 
aumento de peso da matéria seca nos órgãos caulinares e radiciais (PEDROSO-DEMORAES et al., 2011), como descrito neste trabalho.

Com relação às plantas analisadas oriundas do interior do fragmento, os maiores resultados encontrados para CMR, DMR, MFR, CMP, DMP, MFP, CMF, MFF e NMR estão associados à menor intensidade luminosa ocorrida no ambiente. Quando a irradiância é fortemente atenuada, principalmente em formações de dossel contínuo e adensado, como no interior de uma mata, o aparato fotossintético pode se adaptar à menor energia disponível, a fim de manter um balanço positivo de carbono. Nestas condições as espécies podem apresentar maior número de folhas alongadas (CORRÊA, 2004). Quanto a caules e raízes, tais órgãos apresentar-se-ão alongados, mas diametralmente mais espessos, devido à maior disponibilidade de água interna ocorrida pela menor evapotranspiração no mini-habitat, que reflete diretamente nos índices de massa fresca de tais órgãos (LARCHER, 2003; PEDROSO-DE-MORAES, 2002), assim como o encontrado neste trabalho. Resultados semelhantes também foram descritos para a orquídea epífita Catasetum fimbriatum Lindl. em relação ao comprimento foliar e dimensões do pseudobulbo (PEDROSODE-MORAES, 2002). Com relação aos pseudobulbos, maior índice plástico-fenotípico encontra-se relacionado à função armazenadora de água, minerais e carboidratos de tais órgãos (NG; HEW, 2000), que em ambientes com maior disponibilidade hídrica irão absorver água até o máximo de sua capacidade, preparando o vegetal para os períodos estacionais de maior estiagem (LARCHER, 2003; PEDROSO-DE-MORAES, 2002).

Ainda, o maior comprimento médio encontrado para folhas e pseudobulbos reflete-se no deslocamento polar basípeto do ácido 3-indolilacético (IAA). Este hormônio vegetal é sintetizado principalmente no meristema apical e em folhas jovens e é transportado em sentido polarizado, a partir do meristema apical até as extremidades das raízes (AWAD; CASTRO, 1992; TAIZ; ZEIGER, 2004). Esse gradiente longitudinal de auxina controla alguns processos na planta, incluindo o alongamento de órgãos vegetativos, principalmente em ambientes com menor irradiância lumínica (LARCHER, 2003), pois o sistema radicial e a parte aérea de um mesmo vegetal reagem diferentemente à concentração de auxina. A dose ótima para o crescimento da raiz é inferior à dose ótima para o crescimento do caule. A 
raiz, então, é mais sensível ao IAA do que o caule (LARCHER, 2003; TAIZ; ZEIGER, 2004). Como o IAA pode ser degradado na planta por meio da foto-oxidação e pela oxidação enzimática realizada pelo sistema IAA-oxidase (WACHOWICZ; CARVALHO, 2002), ambientes esciófitos geram diminuição nos processos de fotodegradação e translocação de tal molécula e permitem, por aumento de concentração hormonal, maior alongamento de órgãos vegetais (CORRÊA, 2004; LARCHER, 2003).

\section{CONSIDERAÇÕES FINAIS}

As análises morfobiométricas evidenciaram adaptações plástico-fenotípicovegetativas em indivíduos de Oeceoclades maculata viventes em mini-habitats do Arboreto de Essências Nativas. Tais adaptações estão correlacionadas, principalmente às condições lumínicas ocorrentes na borda e no interior do fragmento.

Plantas situadas na borda do fragmento apresentaram maiores índices de plasticidade fenotípica para as variáveis analisadas: massa seca radicial, número médio radicial, massa seca do pseudobulbo, número médio de pseudobulbos, espessura média foliar, largura média foliar e massa seca foliar. As plantas viventes no interior do Arboreto apresentaram maiores valores para comprimento médio radicial, diâmetro médio radicial, massa fresca radicial, comprimento médio do pseudobulbo, diâmetro médio do pseudobulbo, massa fresca do pseudobulbo, comprimento médio foliar, massa fresca foliar e número médio foliar.

\section{REFERÊNCIAS}

ACKERMAN, J. D. Invasive orchids: weeds we hate to love? Lankasteriana, v. 7, p. 19-21, 2007.

ARAÚJO, L. E.; SILVA, K. A.; FERRAZ, E. M. N.; SAMPAIO, E. V. S. B.; SILVA, S. I. Diversidade de herbáceas em microhabitats rochoso, plano e ciliar em uma área de caatinga, Caruaru, PE, Brasil. Acta Botanica Brasilica, v. 19, p. 285-294, 2005. 
AWAD, M.; CASTRO, P. R. C. Introdução à fisiologia vegetal. 2. ed. São Paulo: Biblioteca Rural; Livraria Nobel, 1992.129 p.

BATISTA, J. A. N.; BIANCHETTI, L. B. Lista atualizada das Orchidaceae do Distrito Federal. Acta Botanica Brasilica, v. 17, p. 183-201, 2003.

BATISTA, J. A. N.; BIANCHETTI, L. B.; PELLIZARO, K. F. Orchidaceae da Reserva Ecológica do Guará. Acta Botanica Brasilica, v. 19, p. 221-232, 2005.

BUENO, M. L.; RESENDE, U. M.; GOMES, T. R. Levantamento florístico nas trilhas turísticas da RPPN São Geraldo, Bonito, Mato Grosso do Sul. Revista Brasileira de Biociências, v. 5, p. 189-191, 2007.

CARDOSO, G. L.; LOMÔNACO, C. Variações fenotípicas em Eugenia calycina. Revista Brasileira de Botânica, v. 26, p. 131-140, 2003.

CARRERA, G. C.; BENEDITO, E. F.; CAVENAGHI JÚNIOR, J. A.; SANTOS, M. F.; PEDROSO-DE-MORAES, C.; PIGOSO, A. A.; GASPI, F. O. G. Análise do método de extração e capacidade antioxidante do extrato hidroalcoólico de Oeceoclades maculata Lindl. In: SIMPÓSIO DE PLANTAS MEDICINAIS DE PLANTAS MEDICINAIS DO BRASIL, 19., 2006. Anais... [s.1.; s.n], 2006. p. 36-36.

CEPAGRI. Clima dos municípios paulistas. 2007. Disponível em: < http://www. cpa.unicamp.br/ outras-informacoes/clima_muni_347.html>. Acesso em: 23 abr. 2014.

COHEN, I. M.; ACKERMAN, J. D. Oeceoclades maculata, an alien tropical orchid in a Caribbean rainforest. Annals of Botany, v. 104, p. 557-563, 2009.

CORRÊA, I. C. Plasticidade fenotípica em indivíduos jovens de Aloysia virgata (Ruiz et Pav.) A.L. Juss. - Verbenaceae. 2004. 58 f. Dissertação (Mestrado em Ecologia e Recursos Naturais) - Universidade Federal de São Carlos - UFSCar, São Carlos, São Paulo.

FELDMANN, P.; BARRÉ, N. Atlas des orchidées sauvages de la Guadeloupe. 
Patrimoines naturels, Paris: Muséum National D'Histoire Naturelle, CIRAD, 2001. $228 \mathrm{p}$.

FERREIRA, A. W. C.; LIMA, M. I. S.; PANSARIN, E. R. Orchidaceae na região central de São Paulo, Brasil. Rodriguesia, v. 61, p. 243-259, 2010.

FUTUYMA, D. J.; MORENO, G. The evolution of ecological specialization. Annual Review of Ecology and Systematics, v. 19, p. 207-234, 1988.

FUZETO, A. P.; LOMÔNACO, C. Potencial plástico de Cabralea canjerana subsp. polytricha (Adr. Juss.) Penn. (Meliaceae) e seu papel na formação de ecótipos em área de cerrado e vereda, Uberlândia, MG. Revista Brasileira de Botânica, v. 23, p. 169-176, 2000.

GIANOLI, E. Plasticidad fenotípica adaptativa en plantas. In: CABRERA, E. M. (Ed.). Fisiologia ecológica en plantas. Valparaíso: Universidad Catolica de Valparaíso, 2004. p.13-26

GONZÁLEZ, A.; GIANOLI, E. Morphological plasticity in response to shading in three Convolvulus species of different ecological breath. Acta Oecologica, v. 26, p. 184190, 2004.

KINOSHITA, L. S.; TORRES, R. B.; FORNI-MARTINS, E. R.; SPINELLI, T.; AHN, Y. J.; CONSTÂNCIO, S. S. Composição florística e síndromes de polinização e dispersão da mata do Sítio São Francisco. Acta Botanica Brasilica, v. 20, p. 313-327, 2006.

LARCHER, W. Ecofisiologia vegetal. RIMA, São Carlos, 2003. 592 p.

LIU, H.; PEMBERTON, R. Pollination of an invasive orchid, Cyrtopodium polyphillum (Orchidaceae), by an invasive oil-collecting bee, Centris nitida, in southern Florida. Botany, v. 88, p. 290-295, 2010.

MENINI-NETTO, L.; ALMEIDA, V. R.; FORZZA, R. C. A família Orchidaceae em um fragmento de floresta estacional semidecidual, no município de Barroso, Minas Gerais, Brasil. Lundiana, v. 4, p. 9-24, 2004. 
MORAES, C. P.; ALMEIDA, M. de. Influência climática sobre a plasticidade fenotípica floral de Catasetum fimbriatum LINDLEY. Ciência e Agrotecnologia, v. 28, p. 942$948,2004$.

NG, C. K. Y.; HEW, C. S. Orchid pseudobulbs: "false" bulbs with a genuine importance in orchid growth and survival? Science Horticulture, v. 83, p. 165-172, 2000.

PALACIO-LÓPEZ, K.; RODRÍGUEZ-LÓPEZ, N. Plasticidad fenotípica em Lippia alba (Verbenaceae) em respuesta a la disponibilidade hídrica en dos ambientes lumínicos. Acta Biologica Colombiana, v. 12, p.187-198, 2007.

PANSARIN, E. R.; PANSARIN, L. M. A família Orchidaceae na Serra do Japi, São Paulo, Brasil. Rodriguesia, v. 59, p. 99-111, 2008.

PEDROSO-DE-MORAES, C. Fenologia e anatomia dos órgãos reprodutivos de Catasetum fimbriatum Lindley cultivados sob diferentes intensidades luminosas. 2002. 80 f. Dissertação (Mestrado em Ciências) - Escola Superior de Agricultura Luiz de Queiróz, ESALQ-USP, Piracicaba, São Paulo. 2002.

PEDROSO-DE-MORAES, C.; DOMINGUES, E.; PREZZI, L. E.; SOUZA-LEAL, T.; ZAMBON, R. L.; BRESCANSIN, R. L.; RAMOS, P. A. B. Florística e fitossociologia da família Orchidaceae no Centro de Educação Ambiental "Francisco Mendes", município de Mogi Guaçu, SP, Brasil. Scientia Plena, v. 6, p. 1-5, 2010.

PEDROSO-DE-MORAES, C.; SOUZA-LEAL, T.; PEDRO, N. P.; MARTINI, G. A.; MORO, A. M. AIA no estímulo de brotos laterais em estacas de Dendrobium nobile Lindley (Orchidaceae). Ensaios e Ciência, v. 15, p. 111-119, 2011.

PIGLIUCCI, M. Phenotypic plasticity: beyond nature end nurture. Baltimore: The John Hopkins University Press, 2001. 236 p.

PIVELLO-POMPÉIA, V. R. Exportação de macronutrientes para a atmosfera durante queimadas realizadas no campo-cerrado de Emas (Pirassununga, SP). 1985. 145 f. Dissertação (Mestrado em Ecologia) - Universidade de São Paulo, Instituto de Biociências, São Paulo, 1985. 
SCHULZE, E.; RIBICHAUX, R.; GRACE, J.; RUNDEL, P.; EHLERINGER, J. Plant water balance. BioScience, v. 37, p. 30-37, 1987.

SCHUSTER, H.; PEDROSO-DE-MORAES, C.; SOUZA-LEAL, T.; CALLEGARI-CORREIA, E.; PREZZI, L. E.; DOMINGUES, E.; CANASSA, F. Diversidade de Orchidaceae da fazenda Cantagalo, município de Mogi-Mirim, São Paulo. Revista Brasileira de Biociências, v. 8, p. 242-245, 2010.

SMEKENS, M.; VAN TIENDEREN, M. Genetic variation and plasticity of Plantago coronopus under saline conditions. Acta Oecologica, v. 22, p.187-200, 2001.

STERN, W. L. The long-distance dispersal of Oeceoclades maculata. American Orchid Society Bulletin, v. 57, p. 960-971, 1988.

SULTAN, S.; BAZZAZ, F. Phenotypic plasticity of Polygonum persicaria. Diversity and uniformity in genotypic norms of reaction to light. Evolution, v. 47, p. 1009-1031, 1993.

TAIZ, L.; ZEIGER, E. Fisiologia Vegetal. 3. ed. Porto Alegre: Artmed, 2004. 719p.

THOMPSON, J. D. Phenotypic plasticity as a component of evolutionary change. Trends in Ecology and Evolution, v. 6, p. 246-249, 1991.

VALLADARES, F.; CHICO, J.; ARANDA, I.; BALAGUER, L.; DIZENGREMEL, P.; MANRIQUE, E.; DREYER, E. The greater seedling high-light tolerance of Quercus robar over Fagus sylvatica is linked to greater physiological plasticity. Trees, v. 16, p. 395-403, 2002.

VALLADARES, F.; WRIGHT, S. J.; LASSO, E.; KITAJIMA, K.; PEARCY, R. W. Plastic phenotypic response to light of 16 congeneric shrubs from a panamanian rainforest. Ecology, v. 81, p. 1925-1936, 2000.

VIA, S.; LANDE, R. Genotype-environment interactions and the evolution of phenotypic plasticity. Evolution, v. 39, p. 505-522, 1985. 
VOLTAN, R. B. Q.; FAHL, J. I.; CARELLI, M. L. C. Variação na anatomia foliar de Cafeeiros submetidos a diferentes intensidades luminosas. Revista Brasileira de Fisiologia Vegetal, v. 4, p. 99-105, 1992.

WACHOWICZ, C. M.; CARVALHO, R. I. N. Fisiologia Vegetal: produção e póscolheita. Curitiba: Universitária Champagnat, 2002, 424 p.

WILLIAMS-LINERA, G. Vegetation structure and environmental conditions of forest edges in Panama. Journal of Ecology, v. 78, p. 356-373. 1990.

WRIGHT, S. Evolution of Mendelian populations. Genetics, v. 16, p. 97-159, 1931.

ZOTZ, G.; ANDRADE, J. L. La ecología y la fisiología de las epifitas y las hemiepífitas. In: GUARIGUATA, M.; KATTAN, G. (Ed.) Ecología y conservación de bosques neotropicales. San José: Libro Universitario Regional, 2002. p. 271-296.

Recebido em: 23 de abril de 2014 Aceito em: 20 de agosto de 2014 



\title{
DETERMINAÇÃo DE PESTICIDAS NA ÁGUA E SEDIMENTO DO RIO PIQUIRI
}

\author{
Julio Cesar Rodrigues Azevedo" \\ Eliel R. Rolim de Moura** \\ Mauricius Marques dos Santos ${ }^{* * *}$
}

RESUMO: A qualidade da água e preservação dos ambientes aquáticos assumiu papel fundamental para a sustentabilidade, não só relacionado ao abastecimento, mas também à preservação destes ecossistemas. Os pesticidas fazem parte do grupo de poluentes que podem gerar impactos significativos nos ambientes em que são inseridos, impactos dos quais alguns ainda não são totalmente conhecidos. O objetivo deste estudo foi avaliar amostras de água e sedimento do Rio Piquiri, localizado em uma região de alta produção de trigo, soja e milho, no Estado do Paraná, Brasil. Este rio sofre influência direta da aplicação de pesticidas das lavouras da região. As amostras de água foram extraídas com uma mistura de diclorometano:hexano e as amostras de sedimento com uma mistura de acetona:hexano. Os extratos foram analisados em LC/MS/MS e confirmaram a presença dos herbicidas e fungicidas utilizados na região do estudo conforme dados oficiais de comercialização no período das coletas. Os resultados mais expressivos nas amostras de água foram do herbicida Atrazina $\left(0,030 \mu \mathrm{g} \mathrm{L}^{-1}\right)$ e do fungicida Carbendazim $\left(0,178 \mu \mathrm{g} \mathrm{L}^{-1}\right)$. No sedimento os maiores valores encontrados foram para os fungicidas Carbendazim $\left(26,8 \mu \mathrm{g} \mathrm{L}^{-1}\right)$ e Azoxistrobina $\left(0,712 \mu \mathrm{g} \mathrm{L}^{-1}\right)$, ambos na terceira coleta no ponto de coleta 3. Os resultados obtidos confirmaram a presença de resíduos de herbicidas e fungicidas na água e sedimento do Rio Piquiri, mas as concentrações obtidas não comprometem a potabilidade da água, devido aos valores estarem abaixo do Valor Máximo Permitido.

PALAVRAS-CHAVE: Atrazina; Carbendazim; Fungicidas; Herbicidas; LC/MS/MS.

\section{DETERMINATION OF PESTICIDES IN THE WATER AND SEDIMENT OF THE RIVER PIQUIRI}

\footnotetext{
Doutorado em Ecologia de Ambientes Aquáticos Continentais pela Universidade Estadual de Maringá, Pósdoutorado em Química Analítica na Universidade de Coimbra, Portugal; E-mail: jcrazevedo@hotmail.com

** Mestrando em Ciências e Tecnologia Ambiental pela Universidade Tecnológica Federal do Paraná (UTFPR), Brasil.

*** Discente de Doutorado em Engenharia Ambiental na University of Arizona, Arizona, Estados Unidos.
} 
ABSTRACT: The role of water quality and the preservation of aquatic environments are highly important for sustainability, with special reference to the ecosystems supply and preservation. Pesticides are pollutants that generate significant impacts on the environment, some of which are not totally known. Current research assesses water and sediment samples from the river Piquiri in a region featuring high production of wheat, soybean and corn in the state of Paraná, Brazil. In fact, the river Piquiri is directly affected by pesticides from the surrounding fields. Water samples were extracted with a mixture of hexane : dichloromethane and the sediment samples were extracted with a mixture of acetone : hexane. Extracts were analyzed in LC/MS/ MS and the presence of herbicides and fungicides used in the region under analysis was confirmed. Results of water samples revealed the herbicide atrazine $(0.030 \mu \mathrm{g}$ $\left.\mathrm{L}^{-1}\right)$ and the fungicide Carbendazim $\left(0,178 \mu \mathrm{g} \mathrm{L}^{-1}\right)$. The fungicides Carbendazim $(26.8$ $\left.\mu \mathrm{g} \mathrm{L}^{-1}\right)$ and Azoxistrobina $\left(0.712 \mu \mathrm{g} \mathrm{L}^{-1}\right)$ had the highest rates in the sediments in the third harvest at collection site 3 . Results confirm herbicide and fungicide residues in the water and sediment of the river Piquiri even though concentrations do not compromise water drinkability due to rates below the permitted maximum rate.

KEY WORDS: Atrazine; Carbendazim; Fungicides; Herbicides; LC/MS/MS.

\section{INTRODUÇÃO}

$\mathrm{Na}$ última década cerca de 11 milhões de substâncias químicas estavam registradas no CAS (Chemical Abstracts Service). Neste período, nos Estados Unidos e Japão, cerca de 100.000 compostos foram produzidos deliberadamente e utilizados para diversos fins. Destes, aproximadamente 3.000 compostos são produzidos em larga escala, com quantidades superiores a 500 toneladas por ano. Menos de $45 \%$ das novas substâncias foram submetidos a algum tipo de ensaio toxicológico básico e menos de $10 \%$ foram estudados quanto aos efeitos tóxicos sobre organismos em desenvolvimento (GHISELLI; JARDIM, 2007). Como exemplo podem ser citados os pesticidas, uma vez que muitas fórmulas destes compostos são manipuladas no Brasil e não existem estudos detalhados de muitos destes compostos.

$\mathrm{O}$ uso extensivo de pesticidas visando aumentar a produção agrícola e o intensivo desenvolvimento de novas moléculas aumentou significativamente a quantidade e variedade destes poluentes no ambiente (BAVCON; FRANCO; TREBSE, 
2007; RIBAS; MATSUMURA, 2009; HERNÁNDEZ-ACOSTA et al., 2013), muitas vezes provenientes do desconhecimento dos riscos dos produtos que utilizam e/ou da melhor técnica de sua aplicação.

A exposição ambiental das áreas próximas às lavouras, em função do uso de agroquímicos, depende das propriedades químicas do ingrediente ativo, dos agentes dispersantes da formulação, as doses e formas de aplicação e das características ambientais do local em que a aplicação ocorre (LEWIS et al., 1997; EL BAKOURI et al., 2009).

Os fatores que alteram a mobilidade e persistência dos agroquímicos estão relacionados ao regime de chuvas, intensidade do escoamento das águas superficiais, média do regime térmico, entre outros (NAVARRO et al., 2011). Por sua vez, a vulnerabilidade dos organismos não alvos depende da estrutura espacial e distribuição da biodiversidade da área (ARES, 2003).

A contaminação dos ambientes aquáticos por pesticidas se dá por aplicações intencionais, quando se quer combater uma praga na água como algas, moluscos, mosquitos. Ou por aplicações não intencionais, por diversos mecanismos como precipitação atmosférica, erosão do solo, efluentes industriais, esgoto sanitário, vazamentos durante o processamento, armazenamento e transporte, além das contaminações por pulverização e limpeza de equipamentos (SOLOMON et al., 2010). Independentemente da fonte de contaminação, estas substâncias apresentam efeitos indesejados nos ambientes aquáticos.

O destino dos pesticidas nos sistemas aquáticos é afetado pelas propriedades físicas e químicas da molécula, pela presença de outras substâncias na água, pH, temperatura e interação com o sedimento (SOLOMON et al., 2010).

Devido à sua habilidade de adsorver uma variedade de compostos orgânicos e inorgânicos, e seus subsequentes processos erosivos de entrada nos sistemas aquáticos, os sedimentos de muitos corpos aquáticos têm se tornado verdadeiras fontes de compostos químicos perigosos. Normalmente, estes compostos nocivos se acumulam no sedimento e seu processo de desorção e difusão em águas superficiais tem contribuído para a redução da qualidade da água e dos sedimentos destes ambientes (ZHOU et al., 2011).

Considerando esta realidade, provavelmente, o principal limitante para o desenvolvimento sustentável no início do presente século será a qualidade das águas. 
Primeiramente as águas superficiais e, em um segundo momento, as subterrâneas. Neste contexto, o monitoramento dos pesticidas, especialmente nos ambientes próximos à sua aplicação, constitui um importante indicador ambiental balizador de políticas públicas e ações de controle. Assim, este trabalho teve como objetivo determinar e quantificar a presença de pesticidas em amostras de água e sedimento do Rio Piquiri, Oeste do Estado do Paraná.

\section{MATERIAL E MÉTODOS}

\section{1 ÁREA DE ESTUDO}

A bacia hidrográfica do Rio Piquiri pertence à bacia hidrográfica do Paraná. A nascente do Rio Piquiri fica na Serra do São João, entre os municípios de Turvo e Guarapuava, com altitude inicial de 1.040 metros, chegando a 220 metros em sua foz, na margem do Rio Paraná, percorrendo $660 \mathrm{~km}$, apresentando área total de 24.721 $\mathrm{km}^{2}$ (SEMA, 2010). Os pontos amostrados apresentaram as seguintes coordenadas

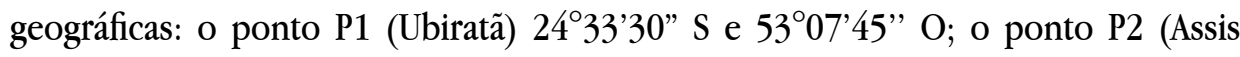

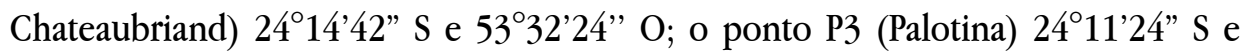

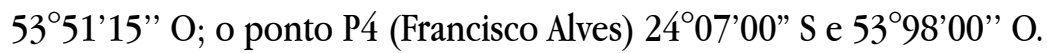

\subsection{ANÁLISE DE PESTICIDAS}

Foi realizada uma varredura qualitativamente de aproximadamente 80 pesticidas: abamectina, alacloro, aldicarbe, aldicarbesulfona, aletrina, ametrina, atrazina, azinfós etílico, azinfós metílico, azoxistrobina, bifentrina, carbofurano, carbosulfano, ciflutrina, cinoxamil, cipermetrinas, ciproconazol, ciprodinil, clofentezina, clomazona, clorfenvinfós, clorpirifós etílico, clorpirifós metílico, cresoxim metílico, carbaril, carbendazina deltametrina, diazinona, diclorvós, difenoconazol, dimetoato, disulfoton, diuron, epoxiconazol, etiona, fluazifop- $p$ butílico, fipronil, fentoato, flufenoxurom, fluometuron, flutriafol, forato, fosalona, fosfolan, fosmete, hexaconazol, hexazinona, hexitiazoxi, imazalil, imidacloprido, indoxacarbe, lambda-cialotrina, linuron, lufenuron, malationa, metalaxil metílico, 
metsulfurom metílico, metiocarbe, metolacloro, metidationa, mevinfós, oxamil, pirimicarbe, pirimifós etílico, profenofós, piriproxifem, procloraz, pirimifós metílico, promecarbe, prometrina, propanil, propargito, propiconazol, propoxur, simazina, tebuconazol, terbufós, tiabendazol, tiacloprido, tiofanato metílico, tiametoxan. Após a separação cromatográfica o analito foi para o espectrômetro de massas. Na câmara de ionização o analito foi transformado no íon principal ou íon precursor. Após a seleção do íon, este colidiu com o gás Hélio, gerando fragmentos iônicos (íons secundários). A varredura foi por monitoramento de múltiplas reações (Multiple Reaction Monitoring) - MRM - empregado para quantificar transição específica de um íon precursor para um íon secundário ( $\mathrm{m} / \mathrm{z}$ - precursor $\mathrm{m} / \mathrm{z}$ secundário).

As coletas de água e sedimento foram realizadas em setembro, outubro e dezembro de 2012 (C1 = set/12; C2 = out/12; e C3 = dez/12). O período das coletas esteve compreendido entre o preparo para o plantio da safra de verão de soja e milho e o fim da colheita do trigo na região.

Para a coleta das amostras de água foi utilizada garrafa de Van Dorn a 1,5 metros de profundidade em cinco subpontos equidistantes em aproximadamente 2 metros. Em cada subponto foi coletado 1 litro de água em garrafa de vidro âmbar, perfazendo um total de 5 litros que foram homogeneizados. Para a coleta das amostras de sedimento foi utilizada a draga de Petersen modificada. As coletas foram realizadas em três subpontos, sendo coletados aproximadamente 400 gramas de sedimento, perfazendo um total de 1,2 quilogramas, que foi homogeneizado (EMBRAPA, 2006).

As amostras de água e sedimento foram coletadas e preservadas a $4^{\circ} \mathrm{C}$ em caixas térmicas e, posteriormente, foram congeladas e armazenadas em freezer até o momento da extração. Ambas foram coletadas em pontos próximos à margem buscando evitar a ação da força hidráulica. O conceito de combinação de amostras simples e a limpeza dos frascos seguiram as recomendações da Embrapa (2006).

\subsection{EXTRAÇÃO E ANÁLISE CROMATOGRÁFICA}

Nas amostras de água, as análises de pesticidas foram realizadas em 1 litro de amostra de água filtrado em membrana de $13 \mathrm{~cm}$ de diâmetro e 0,22 $\mu \mathrm{m}$ de PVDF 